University of Nebraska - Lincoln

DigitalCommons@University of Nebraska - Lincoln

$10-22-2004$

\title{
Hybrid atomistic-coarse-grained treatment of thin-film lubrication.
}

II

Z.-B. Wu

University of Nebraska-Lincoln

Dennis J. Diestler

University of Nebraska-Lincoln, ddiestler1@unl.edu

Xiao Cheng Zeng

University of Nebraska-Lincoln, xzeng1@unl.edu

Follow this and additional works at: https://digitalcommons.unl.edu/chemzeng

Part of the Chemistry Commons

Wu, Z.-B.; Diestler, Dennis J.; and Zeng, Xiao Cheng, "Hybrid atomistic-coarse-grained treatment of thinfilm lubrication. II" (2004). Xiao Cheng Zeng Publications. 25.

https://digitalcommons.unl.edu/chemzeng/25

This Article is brought to you for free and open access by the Published Research - Department of Chemistry at DigitalCommons@University of Nebraska - Lincoln. It has been accepted for inclusion in Xiao Cheng Zeng Publications by an authorized administrator of DigitalCommons@University of Nebraska - Lincoln. 


\title{
Hybrid atomistic-coarse-grained treatment of thin-film lubrication. II
}

\author{
Z.-B. Wu \\ Department of Chemistry, University of Nebraska-Lincoln, Lincoln, Nebraska 68588 \\ and LNM, Institute of Mechanics, Academia Sinica, Beijing 100080, China \\ D. J. Diestler \\ Department of Agronomy and Horticulture, University of Nebraska-Lincoln, Lincoln, Nebraska 68583 \\ X. C. Zeng \\ Department of Chemistry, University of Nebraska-Lincoln, Lincoln, Nebraska 68588
}

(Received 5 February 2004; accepted 19 July 2004)

\begin{abstract}
A new hybrid atomistic-coarse-grained (HACG) treatment of reversible processes in multiple-scale systems involving fluid-solid interfaces was tested through isothermal-isobaric Monte Carlo simulations of the quasistatic shearing of a model two-dimensional lubricated contact comprising two planar Lennard-Jones solid substrates that sandwich a softer Lennard-Jones film. Shear-stress profiles (plots of shear stress $T_{y x}$ versus lateral displacement of the substrates) obtained by the HACG technique, which combines an atomistic description of the interfacial region with a continuum description of regions well removed from the interface, are compared with "exact" profiles (obtained by treating the whole system at the atomic scale) for a selection of thermodynamic states that correspond to systematic variations of temperature, load (normal stress), film-substrate coupling strength, and film thickness. The HACG profiles are in excellent agreement overall with the exact ones. The HACG scheme provides a reliable description of quasistatic shearing under a wide range of conditions. It is demonstrated that the elastic response of the remote regions of the substrates can have a significant impact on the static friction profile (plot of maximum magnitude of $T_{y x}$ versus load). (C) 2004 American Institute of Physics. [DOI: 10.1063/1.1792154]
\end{abstract}

\section{INTRODUCTION}

This article is the second of a pair devoted to the development and testing of a treatment of reversible processes in multiple-scale systems involving fluid-solid interfaces. The prototypal tribological system consists of two planar solid substrates separated by a thin fluid film. Relative sliding of the substrates entails the coupling of various processes on spatial (and temporal) scales ranging from atomic to macroscopic. In the vicinity of the interface film molecules, as well as substrate atoms, can execute large-amplitude (diffusive) motions, the details of which are expected to exert a strong influence on the course of sliding. To describe the manybody motion on the atomic scale with the necessary precision, one must keep track of individual atoms in the film and in the "near" regions of the substrates (i.e., in the several layers of substrate atoms that are conterminous with the film). However, to follow the motion of all atoms in the system is practically impossible. Nevertheless, the response of the "far" regions (i.e., the regions of the substrates that are remote from the interface) can significantly influence the course of sliding, as is demonstrated by the results presented below (see Sec. IV C). Since the far regions are only slightly strained (that is, the atoms of the far regions oscillate with small amplitude around their equilibrium positions, which are displaced only slightly in sliding), they can be adequately handled at the continuum level. That is, one can coarse grain the far regions, thereby suppressing details of the atomic motion, which are (presumably) irrelevant to the process of interest, namely, sliding in the present instance. It remains to merge the atomic description necessary for the film and near regions with the coarse-grained description sufficient for the far regions in a reliable global description.

The evolution of defects in solids bears a close relationship to sliding in that it involves the relative motion of parts of the same solid. Irregular large-amplitude atomic motions near the defect must be coupled to smooth, small displacements in regions remote from the defect. A number of techniques combining atomistic and continuum descriptions of the behavior of defects in solids have been put forth since the early 1990 's ${ }^{1-14}$ and these have been exhaustively reviewed in the last couple of years. ${ }^{15-19}$ Of particular relevance is the quasicontinuum (QC) method, ${ }^{3,15,16,19}$ which we have adapted in formulating our new hybrid atomistic-coarsegrained description of the prototypal tribological system. The essential idea behind the QC technique is to coarse grain the entire atomic lattice of the solid by covering it with a finiteelement (FE) mesh. Thereby all atoms are explicitly eliminated, and only the nodes of the FE mesh remain as quasiparticles. The effective interaction between the quasiparticles is mediated by the original atoms, which move in unison with the nodes, as dictated by the FE interpolating functions. ${ }^{3,20}$ In regions remote from the defect, where the lattice is essentially perfect, large (mesoscale) elements are used, whereas in the vicinity of the defect, where the lattice is highly irregular, atomic-scale elements are used. Hence, there is a gradation in the size of elements from atomic near the defect to mesoscopic far from the defect. In this manner multiple scales are seamlessly melded. The static configura- 
tion of the nodes is determined by minimizing the effective potential energy (expressed as a sum of elemental contributions) under prescribed boundary conditions. This minimum principle implicitly assumes zero temperature $(T=0 \mathrm{~K})$. There is no motion in real time. The (metastable) defect evolves quasistatically as the boundary conditions are incrementally altered to mimic variations in applied strains or stresses.

To bridge the gap between atomic and macroscopic scales in tribological systems, which involve relative motion at fluid-solid interfaces, we are pursuing a hybrid atomisticcoarse-grained (HACG) treatment based on extensions of the QC method. In the first paper, ${ }^{21}$ hereafter referred to as I, we developed the formalism. The key idea is to coarse grain the (prototypal) system only partially. The film and near regions of the substrates are described explicitly at the atomic scale, that is, every atom in the film and near regions is tracked. Only the far regions are blanketed by (finite-element) coarsegraining (CG) meshes in the same fashion as in the original QC procedure. ${ }^{3}$ The coarse-grained far regions are dynamically constrained in that the non-nodal atoms underlying the CG mesh are forced to move in concert with the nodes according to the finite-element interpolation functions. ${ }^{20}$ Specifically, the underlying lattice is assumed to deform homogeneously in response to displacements of the nodes. The HACG formalism yields an effective Hamiltonian for the real atoms of the film and near regions coupled to the pseudoatoms (nodes) of the far regions.

Sliding is treated as a quasistatic (reversible) process. Here again time is irrelevant. The Gibbs potential of the system is minimized for a succession of states specified by the relative lateral displacement (register) of the substrates under a fixed normal load that presses the substrates together and a fixed temperature. In practice this is accomplished by means of an isothermal-isobaric ensemble Monte Carlo simulation. Thermomechanical properties are computed as ensemble averages. Sliding is characterized principally by the shear-stress profile, which is simply a plot of shear stress as a function of register.

As "proof of principle" in I we presented the results of simulations of a two-dimensional (2D) model lubricated contact: Lennard-Jones $(12,6)$ substrates sandwiching a Lennard-Jones monolayer film. We observe excellent agreement overall between the HACG shear-stress profile and its fully atomistic counterpart (i.e., the profile obtained by treating all atoms of the system explicitly), which we take to be the true and "exact" profile.

Since the simulations reported in I are restricted to a single thermodynamic state (specified by temperature, normal load, strength of film-substrate coupling, and thickness of film), the domain of reliability of the HACG technique is unknown. The main purpose of this article is to present the results of additional simulations in which the state parameters are systematically varied. For computational economy we continue to employ the 2D model contact of modest size. Our objective is to demonstrate the validity of the HACG method over a four-dimensional parameter space. For this purpose we need to generate both the HACG and exact (fully atomistic) shear-stress profiles for each set of fixed param-

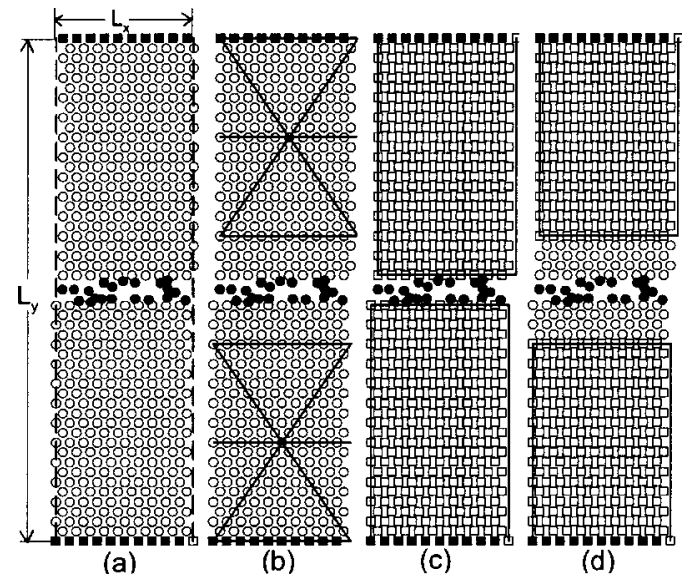

FIG. 1. Schematics of several versions of the 2D model lubricated contact: (a) fully atomistic; (b) partially coarse-grained; (c) wholly rigid substrate; (d) atomistic four-layer near region. Two-layer film $\left(N_{f}=20\right)$ is depicted with atoms (filled circles) in random initial configuration. Register is shown as $\alpha=0.5\left(\alpha^{\prime}=0\right)$. Periodic boundary conditions are applied in $x$ direction. Period, indicated by vertical dashed lines in (a), is $L_{x}=10 a$. Top (bottom) wall atoms are represented by filled squares. In (c) and (d) atoms in atomistic near regions are represented by open circles; atoms in rigid far regions by open squares.

eters. The construction of each profile in turn necessitates simulations at a succession of registers. Actually, the register itself counts as another state parameter. This survey over the thermodynamic state space represents an enormous amount of computing and we must therefore reduce the computational burden even further by exploring only limited subspaces of the whole parameter space.

\section{DESCRIPTION OF THE MODEL}

Since the model was detailed in I, we summarize here only its essential features. Figure 1 displays schematics of the atomistic (a) and coarse-grained (b) versions of the 2D model, as well as variants that are chosen to assess the importance of elasticity of the substrates (see Sec. IV C). Periodic boundary conditions are enforced in the $x$ direction. The top layer of the upper substrate (top wall) and the bottom layer of the lower substrate (bottom wall) are rigid. Shearing is effected by moving the top wall while keeping the bottom one fixed. The lateral alignment (in the $x$ direction) of the walls is specified by the register $\alpha$, defined by $x^{u}=x^{l}+\alpha a$, where $u$ and $l$ denote the respective $x$ coordinates of corresponding atoms in the top and bottom walls and $a$ is the lattice constant of the (rigid) substrate.

The potential energy is expressed as a sum of shifted Lennard-Jones $(12,6)$ interactions $\left(u_{f f}, u_{f s}, u_{s s}\right)$ between pairs of atoms, where the subscripts $f$ and $s$ identify an atom as either a film atom or a substrate atom. The effective diameter $\sigma$ and range $r_{c}$ are the same for all possible pairs. Only the depth of the attractive well $\left(\boldsymbol{\epsilon}_{f f}, \boldsymbol{\epsilon}_{f s}, \boldsymbol{\epsilon}_{s s}\right)$ depends on the composition of the pair.

The CG mesh consists of congruent equilateral triangles in the initial reference configuration depicted in Fig. 1(b). We assume that elements deform homogeneously under displacements of the nodes. Under the dynamic constraint that non-nodal atoms move in lockstep with the nodes, the origi- 
TABLE I. Parameters of the simulation. Reduced dimensionless units are based on the Lennard-Jones parameters $\left(\sigma, \epsilon_{s s}\right)$ of the substrate, as explained in text.
Number of fluid atoms for an $n_{l}$-layer film Number of wall atoms

Total number of substrate atoms

Number of near-region atoms

Number of far-region atoms

Number of elements

Number of nodes

Lattice constant of substrate

Width of contact

Register increment

Substrate-substrate Lennard-Jones well depth

Film-film Lennard-Jones well depth

Cutoff radius

Total number of Monte Carlo cycles

$$
\begin{aligned}
& N_{f}=10 n_{l} \\
& N_{w}=2 \times 10=20 \\
& N_{s}=2 \times 240=480 \\
& N_{s}^{\prime}=2 \times 40=80 \\
& N_{s}^{\prime \prime}=2 \times 200=400 \\
& N_{e}=2 \times 4=8 \\
& N_{n}=2 \times 2=4 \\
& a=\sqrt{(2 / \sqrt{3})}=1.075 \\
& L_{x}=10 a \\
& \Delta \alpha=0.05 \\
& \epsilon_{s s}=1 \\
& \epsilon_{f f}=1 / 9 \\
& r_{c}=2.5 \\
& M=10^{6}
\end{aligned}
$$

$\left.N_{f}, \kappa\right), \kappa$ (at fixed $T, T_{y y}, N_{f}$ ), and $N_{f}$ (at fixed $\left.T, T_{y y}, \kappa\right)$ on the shear-stress profile. Our purpose is to ascertain the ranges of these thermodynamic-state parameters over which the HACG scheme is valid. To this end we compare HACG shear-stress profiles with the corresponding exact (i.e., fully atomistic) profiles.

\section{RESULTS AND DISCUSSION}

Since all shear-stress profiles possess a similar nature, it is worthwhile to summarize their common features before presenting the specific results. For convenience we "quantize" $N_{f}$ by setting $N_{f}=10 n_{l}$, where $n_{l}$ stands for the number of layers of atoms in the film. Although the initial configuration of film atoms is random, as depicted in Fig. 1 for the special case $n_{l}=2$, after equilibration the film atoms assume a solidlike order induced by the substrate surfaces, which function as templates for the growth of the film. Film atoms "adsorbed" on the surface fit neatly into the interstices to form an ordered epitaxial layer. ${ }^{22}$ If $n_{l}=1\left(N_{f}=10\right)$, a single layer just fits between the two substrates when $\alpha=0$. If $n_{l}=2\left(N_{f}=20\right)$, then two adsorbed layers are present and they mesh precisely when $\alpha=0.5$ (or -0.5 ), that is when the top wall is moved to the right (or left) by one-half a lattice constant, as illustrated in Fig. 1. In general, if $n_{l}$ is odd, then the layers mesh when $\alpha$ is even half integral $(\alpha=0, \pm 1$, $\pm 2, \ldots)$, whereas, if $n_{l}$ is even, then they mesh when $\alpha$ is odd half integral $(\alpha= \pm 0.5, \pm 1.5, \ldots)$. In other words, (sufficiently thin) films containing odd (even) numbers of layers are solidlike at even (odd) half-integral registers. Under these conditions the net lateral component of the force on the walls vanishes (i.e., $T_{y x}=0$ ), as indicated in Fig. 2(a), for example.

On account of the template effect, for convenience we implicitly shift the register by 0.5 in the shear-stress profiles for films containing even numbers of layers. Thus, for the two- and four-layer cases (see Fig. 6) the shifted register is $\alpha^{\prime}=\alpha-0.5$. A cursory survey of the several shear-stress profiles displayed in Figs. 2 and $4-8$ reveals characteristic features that were rationalized in I for the monolayer film. The profile is periodic in $\alpha$ and the period is 1 . Over the range $0<\alpha<1$ the profile is antisymmetric about $\alpha=0.5$, for odd $n_{l}$. Likewise, for even $n_{l}$ the profile is antisymmetric about $\alpha^{\prime}=0$ in the range $-0.5<\alpha^{\prime}<0.5$. These symmetries are a direct reflection of the symmetries inherent in the atomic structure of the substrates. Strictly speaking, however, we should note that the partially coarse-grained system is truly periodic in $\alpha$ only on the scale of the dimension of the elements. For the particular CG mesh employed in the present study [see Fig. 1(b)], the true period is $L_{x}$. Nevertheless, because the mesh is sufficiently far removed from the interface, the symmetry character of the profiles is dominated by the local atomic-scale symmetry of the near regions.

The profile comprises "elastic" (stick) regions $(0<\alpha$ $<0.4$ and $0.6<\alpha<1$ for odd $n_{l} ;-0.5<\alpha^{\prime}<-0.1$ and $0.1<\alpha^{\prime}<0.5$ for even $n_{l}$ ). These regions are separated by a "transition" (slip) region $\left(0.4<\alpha<0.6\right.$, for odd $n_{l}$; $-0.1<\alpha^{\prime}<0.1$, for even $n_{l}$ ). As the walls are misaligned (by increasing $\alpha$ gradually from $\alpha=0$ for "odd" films or by in- 

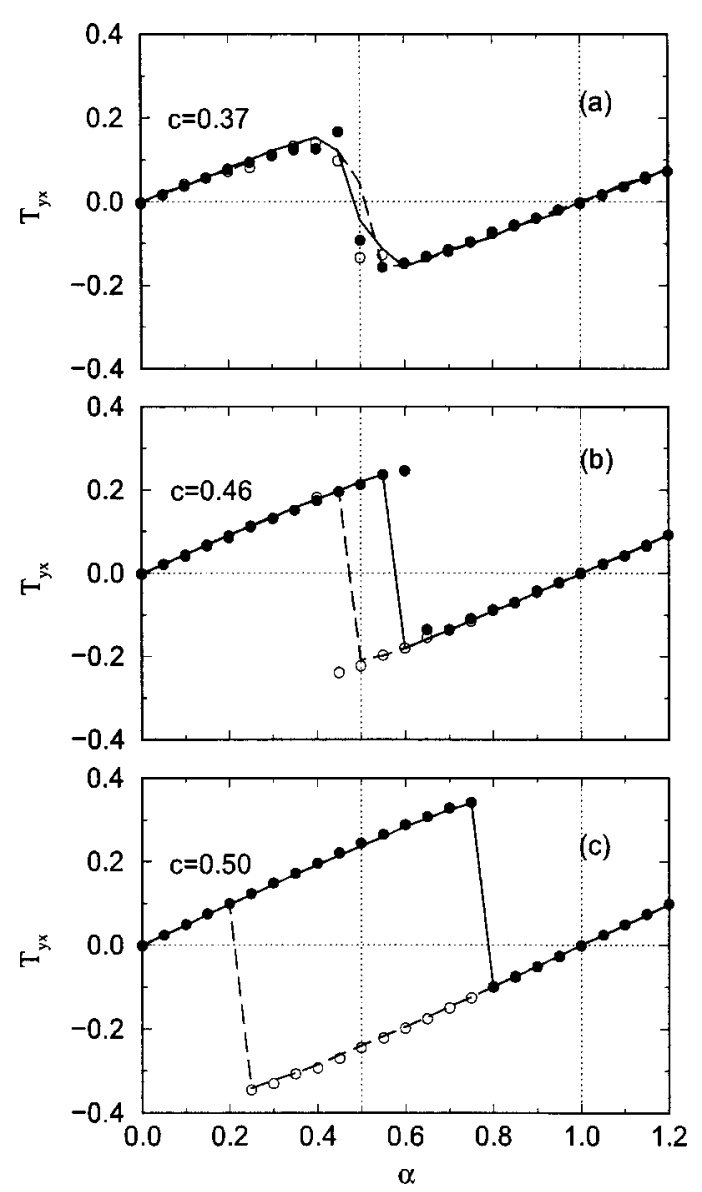

FIG. 2. Shear-stress profiles ( $T_{y x}$ vs register $\alpha$ ) for model contact with monolayer film at several temperatures and fixed $T_{y y}=-0.1, \kappa=1 / 3, n_{l}$ =1. (a) $T=0.1$; (b) $T=0.05$; (c) $T=0.01$. Fully atomistic profile (forward, filled circles; reverse, open circles); HACG profile (forward, solid line; reverse, dashed line). Slope of profile in elastic region (effective thermal elastic coefficient associated with shearing), based on fully atomistic data, is denoted by $c$.

creasing $\alpha^{\prime}$ from $\alpha^{\prime}=-0.5$ for "even" films), a restoring force tends to push them back into alignment. To hold the walls out of alignment a force equal to the shear force, which is the product of the shear stress $T_{y x}$ times the length of the contact, must be applied in the positive $x$ direction. This force increases approximately linearly over the elastic region $\left(0<\alpha<0.4\right.$ for odd films; $-0.5<\alpha^{\prime}<-0.1$ for even films). That is, the contact behaves as if it were a linear elastic solid. We take the slope of the profile in the elastic region to be the "thermal elastic coefficient" $c$ associated with shearing. As $\alpha$ $\left(\alpha^{\prime}\right)$ continues to increase into the transition region, the layers of the film become progressively more disordered and less able to mesh. Directly at the register of maximum misalignment $\left(\alpha=0.5\right.$ for odd $n_{l} ; \alpha^{\prime}=0$ for even $\left.n_{l}\right)$, the layers cannot mesh. Even so, by virtue of the symmetry of the potential field due to the rigid walls, the film atoms should be so distributed that the sheer stress vanishes on average. The actual profiles indicate that this is only approximately true. Nevertheless, it is clear that $T_{y x}$ undergoes an abrupt change of sign in the vicinity of this register of maximum misalignment. If the upper wall is displaced in either direction $(+x$ or $-x$ ) from $\alpha=0.5$ (for odd $n_{l}$ ) or $\alpha^{\prime}=0$ (for even $n_{l}$ ), then a force acts on the wall in the same direction. Therefore, if no external force is applied to the upper wall to keep the register fixed at $\alpha=0.5$ (for odd $n_{l}$ ) or $\alpha^{\prime}=0$ (for even $n_{l}$ ), it will be spontaneously displaced by a slight fluctuation in the register. The system is in a state of unstable equilibrium and tends to lower its free energy by altering the register so that the fluid layers can mesh.

\section{A. Monolayer films}

\section{Thermal effects}

Figure 2 displays shear-stress profiles for the model contact at several temperatures for the weakest film-substrate coupling $(\kappa=1 / 3)$ and the lowest load $\left(T_{y y}=-0.1\right)$. The profile at the highest temperature $(T=0.1)$ [Fig. 2(a)] was obtained and its character analyzed exhaustively in I. We note that when $T$ exceeds about 0.12 surface melting and dissolution of the substrates ensues. For the present purpose we therefore restrict the temperature to lie below $T=0.1$.

The structure of the shear-stress profile was rationalized on an atomic basis in I. The essential idea is that the film molecules are constrained to cages effectively formed by the surrounding atoms of the substrates. As $\alpha$ increases from $\alpha=0$, the cages become increasingly constricted. As a consequence, the mean positions of the caged film molecules skew so that the net shear force rises approximately linearly (in the range $0<\alpha<0.4$ ). The increasing constriction of the cages is also reflected in the mean separation between the top and bottom walls $\left\langle L_{y}\right\rangle$, which is plotted in Fig. 3(a) for the state under consideration presently $\left(T=0.1, T_{y y}=-0.1\right.$, $\left.\kappa=1 / 3, n_{l}=1\right)$. As the cages become more constricted with increasing $\alpha$ in the range $0<\alpha<0.5$, the net force acting on the upper substrate tends to increase. In response the top wall shifts in the positive $y$ direction in order to maintain the prescribed load. $\left\langle L_{y}\right\rangle$ increases very gradually with increasing $\alpha$ and the rate of increase itself increases when the register exceeds about $\alpha=0.4$. From Fig. 2(a) we also see that $T_{y x}$ begins to decline precipitously beyond this register. "Snapshots" of atomic configurations, which are not presented for reasons of economy, indicate that atoms in the upper substrate are beginning to slip over the caged film molecules to occupy mean positions that lie nearly over the voids between adjacent atoms in the lower substrate. The old cages are shrinking to create new ones. Yet the distribution of film molecules remains asymmetric so that the net force on the top wall persists, although it is weaker. At $\alpha=0.5$ there are essentially twice as many cages of half the size as there are at $\alpha=0$ and these cages are equally likely, statistically speaking, to be occupied by a film molecule. Hence, the net transverse force on the top wall vanishes. Nevertheless, since the cages are in their most constricted condition at $\alpha=0.5$, the distance between the walls is maximum [see Fig. $3(\mathrm{a})]$.

As $\alpha$ continues to increase in the range $0.5<\alpha<1$, the sequence of changes described above for the range $0<\alpha<0.5$ occurs in reverse. In the latter half of the unit interval the mean positions of the caged film molecules are skewed in the opposite direction from that in which they were skewed in the first half $(0<\alpha<0.5)$, so that the sign of $T_{y x}$ at $\alpha$ in the second half is the negative of the sign of $T_{y x}$ at $1-\alpha$ in the 

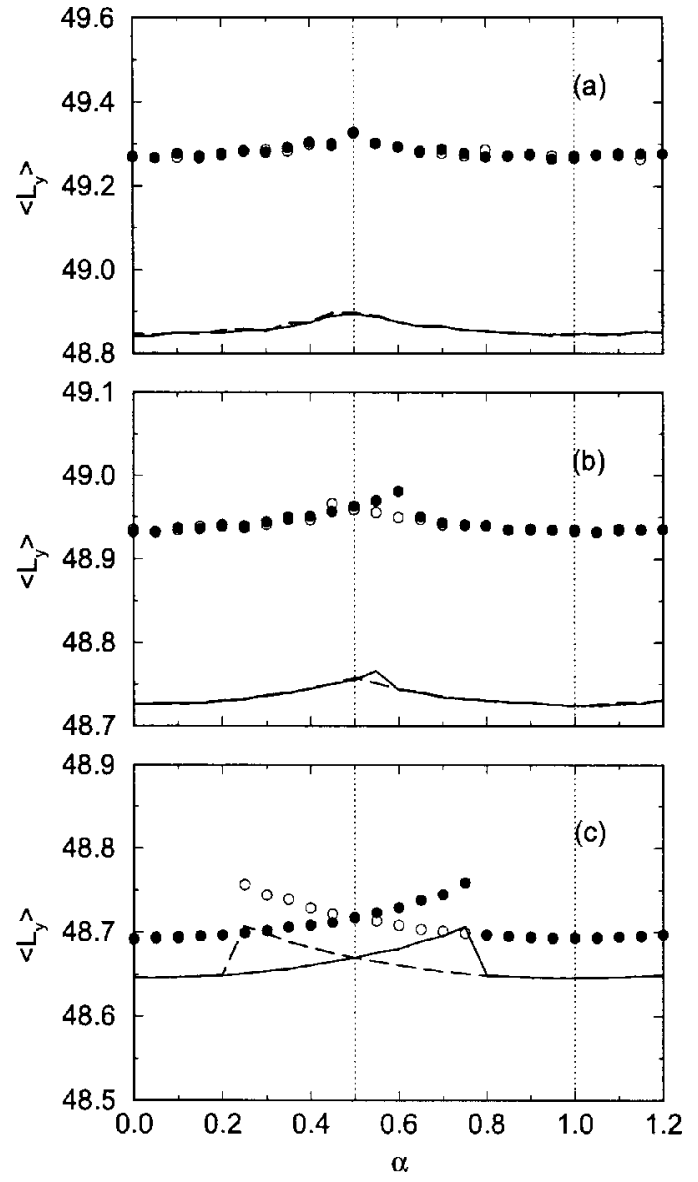

FIG. 3. Mean separation between walls $\left\langle L_{y}\right\rangle$ vs register $\alpha$ for model contact with monolayer film at several temperatures and fixed $T_{y y}=-0.1, \kappa=1 / 3$, $n_{l}=1$. (a) $T=0.1$; (b) $T=0.05$; (c) $T=0.01$. Notation as in Fig. 2 .

first half. Finally at $\alpha=1$, the walls are again in perfect register and the transverse force on the top wall vanishes. As $\alpha$ proceeds to increase from $\alpha=1$, the above described sequence therefore repeats itself. Likewise, because the system is assumed to be in thermodynamic equilibrium, decreasing $\alpha$ from $\alpha=1$ to $\alpha=0$ should generate a "reverse" profile that coincides precisely with the "forward" profile. The plots in Fig. 2(a) appear to confirm this.

Although the forward and reverse HACG profiles are in very good agreement with the corresponding fully atomistic profiles in the elastic regions, there are noticeable discrepancies in the transition region. Indeed, we observe that the forward and reverse profiles for both the atomistic and HACG simulations disagree. In other words, the profile in the transition region depends on the direction of shearing. The system exhibits hysteresis. This is understandable in light of the structural changes the effective cages undergo, as described above. As the original cages shrink to give rise to new, more constricted ones, the film molecules become more constrained. That is, the potential energy barriers confining the film molecules to their respective cages rise. Likewise, effective barriers to the slipping of upper-substrate atoms over the constrained film molecules increase. Hence, transitions of film molecules among the cages, as well as "slipping" transitions of upper-substrate atoms, which should take place as the stochastic MC trajectory evolves, in order that configu- rational space be correctly sampled for the given equilibrium state, become less frequent. The consequence is that the configuration tends to get stuck. This is reflected in the ensemble average, which pertains to a biased configurational distribution rather than to that dictated by the Boltzmann distribution. Increasing the number $M$ of MC cycles should reduce the degree of hysteresis, although we have attempted this tack to no avail. From another perspective, we note that such hysteresis is of course observed in real systems, on which measurements must be made in finite time. If the time of measurement is too short, the system may not achieve a state of equilibrium, perhaps on account of potential barriers of the sort described above. That is, the hysteresis we observe in the model system has a counterpart in nature and is therefore intrinsically interesting.

One intuits that the degree of hysteresis should be greater for thermodynamic states in which the barriers are higher. Specifically, we surmise that the degree of hysteresis (as measured by the breadth of the hysteresis loop) should increase with decreasing $T$, at fixed $T_{y y}, N_{f}$, and $\kappa$. Both shear-stress and separation profiles shown in Figs. 2 and 3, respectively, bear this out. The hysteresis loop broadens considerably as the temperature decreases toward $T=0$. It is perhaps noteworthy that the HACG profile agrees so well with the fully atomistic one. This observation lends support to the atomic-scale "interfacial" mechanism of hysteresis put forward above.

The separation profiles in Fig. 3 show that the mean distance between the walls is slightly greater for the fully atomistic simulation than for the HACG simulation. This is reasonable in view of the dynamic constraints on the atoms in the coarse-grained far regions. The underlying non-nodal atoms are not free to respond individually to the random thermal motions of their neighbors, but rather they are forced to move in lockstep with the nodes. Hence, the coarsegrained far regions are more compressed than their fully atomistic counterparts.

Scrutiny of the shear-stress profiles in Fig. 2 reveals that the effective elastic coefficient $c$ associated with shearing (i.e., the slope of the shear-stress profile) increases gradually as the temperature decreases. The amplitude of random thermal displacements of atoms from their equilibrium positions wanes as $T$ decreases. As a result, the effective force constant associated with atomic displacements increases. Accordingly, as the temperature is lowered, the force that must be applied to the top wall in order to sustain a given register must be increased. By similar reasoning one can rationalize the decrease in $\left\langle L_{y}\right\rangle$ with decreasing $T$, as well as the decline in the discrepancy between the HACG and fully atomistic separation profiles (see Fig. 3).

\section{Effects of load (normal stress)}

In Fig. 4 are shown shear-stress profiles for the model contact under several loads with the temperature fixed at $T$ $=0.1$ and the film-substrate coupling at $\kappa=1 / 3$. We note that for these values of $T$ and $\kappa$ the film tends to become gaslike and the shear-stress profile to flatten if the load is lighter than $T_{y y}=-0.1$. We therefore consider only heavier loads. We see in Fig. 4 that the degree of hysteresis increases with load. 


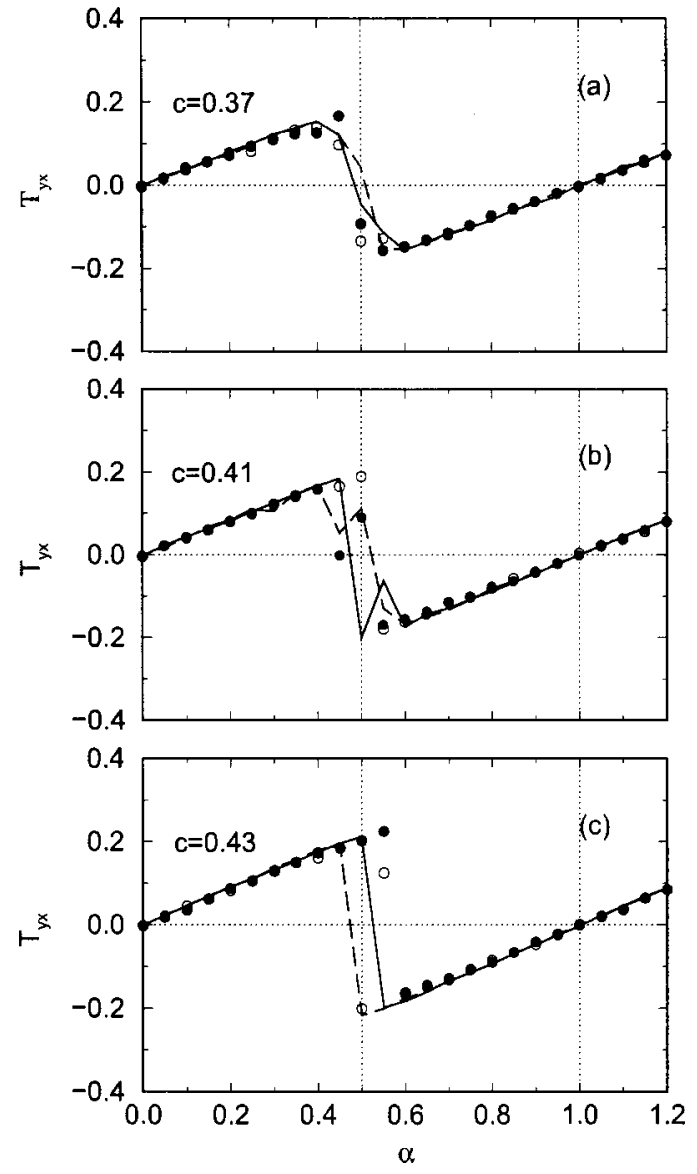

FIG. 4. Shear-stress profiles for model contact with monolayer film at several loads and fixed $T=0.1, \kappa=1 / 3$, and $n_{l}=1$. (a) $T_{y y}=-0.1$; (b) $T_{y y}=$ -0.2 ; (c) $T_{y y}=-0.3$. Notation as in Fig. 2 .

This is expected, since the cages become more constricted as the load increases. Again, the HACG and fully atomistic profiles are in very good agreement overall. And again, as expected, the effective elastic coefficient increases with load, although at a lower rate than that observed in Fig. 2.

\section{Influence of film-substrate coupling strength}

In Fig. 5 we exhibit shear-stress profiles for the model contact for several values of the coupling constant $\kappa$ at fixed $T=0.1$ and fixed $T_{y y}=-0.1$. For the lowest coupling constant $(\kappa=1 / 3)$ the film-substrate Lennard-Jones well depth $\epsilon_{f s}=1 / 9$ is equal to the fixed film-film well depth (see Table I), that is the strength of attraction between a film molecule and a substrate atom is equal to that between two film molecules. However, at the greater values of $\kappa$ considered here $(\kappa=1 / 2$, and $\kappa=1)$ film molecules are preferentially attracted to substrate atoms. The consequence is that they tend to get locked into their respective cages and to move in concert with the substrate atoms as the register changes. In other words, as the coupling constant increases, the film coats the substrate. This behavior, evident in snapshots of configurations, explains why the degree of hysteresis increases markedly with increasing $\kappa$. In particular, for the case $\kappa=1$ [Fig. 5 (c)] the elastic region of the shear-stress profile is about twice as wide as that of the "reference" profile [Fig. 5(a)], where shearing is nearly reversible. On the forward path the
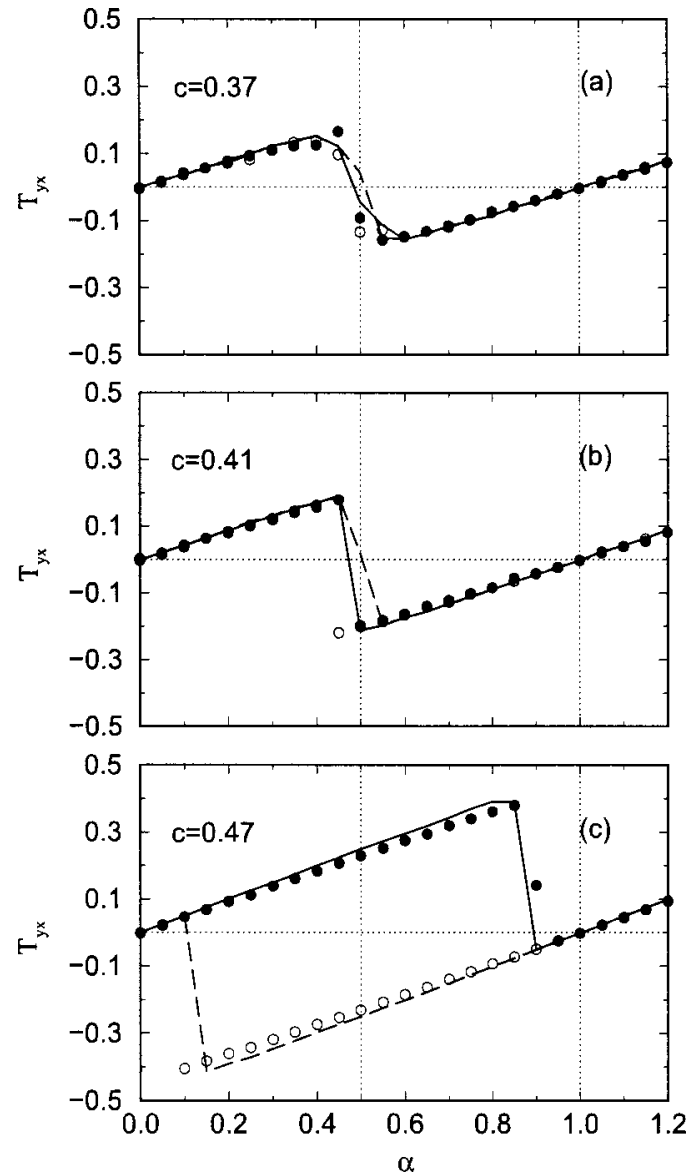

FIG. 5. Shear-stress profiles for model contact with monolayer film at several coupling strengths $\kappa$ and fixed $T=0.1, T_{y y}=-0.1$, and $n_{l}=1$. (a) $\kappa=1 / 3 ; \kappa=1 / 2 ; \kappa=1$. Notation as in Fig. 2 .

contact behaves as if it were a linear elastic solid until a critical register of about $\alpha=0.85$ is reached, whereupon it "snaps" over a very narrow transition region. Symmetric behavior is observed on the reverse path. Only in the transition regions do snapshots reveal any significant fluctuation in the positions of film atoms. It is gratifying that even in this extreme circumstance the HACG profile compares favorably overall with the exact one, although the effective elastic coefficient of the HACG profile is slightly greater (in the hysteretic region of the profile) than that of the fully atomistic one.

Finally, we note that, in keeping with intuition, the effective thermal elastic coefficient increases with increasing $\kappa$ with all other state variables fixed.

\section{Dependence of thermal and load effects on film- substrate coupling strength}

To this juncture we have implicitly regarded the state $T$ $=0.1, T_{y y}=-0.1, \kappa=1 / 3$ as a reference and compared HACG with fully atomistic shear-stress profiles obtained by holding two of these parameters fixed while varying the third. We have performed several additional sets of simulations in order to determine the effects of increasing the filmsubstrate coupling strength on variations of the profiles with temperature and load. For lack of space we do not present the results graphically, but merely summarize them as follows. 
We have examined thermal effects for the greater values of the coupling constant $(\kappa=1 / 2,1)$, keeping the load fixed at $T_{y y}=-0.1$. For the case $\kappa=1 / 2$ the extent of hysteresis is considerably greater than for the weakest coupling strength $\kappa=1 / 3$, yet HACG and fully atomistic profiles are in good agreement. For the case $\kappa=1$ there is a broad hysteresis loop even at the highest temperature $T=0.1$ (unlike the case $\kappa=1 /$ 3 ), but HACG and fully atomistic profiles still agree well. At the lower temperatures $(T=0.05$ and 0.01$)$ the loop extends beyond the range of registers considered $(0<\alpha<1.2)$.

We also looked at the influence of load on the structure of the shear-stress profile at the larger coupling constants and with the temperature fixed at $T=0.1$. For $\kappa=1 / 2$ the hysteresis loop is relatively narrow and broadens only slightly with increasing $T_{y y}$. The HACG and fully atomistic profiles continue to agree well overall. For the strongest coupling $(\kappa=1)$ the hysteresis loop dominates the entire profile in the range $0.2<\alpha<0.9$ at all loads studied. At the lightest loads ( $T_{y y}$ $=-0.1,-0.2)$ the hybrid and fully atomistic profiles agree well, except that the elastic coefficient for the hybrid profile is greater than that of the fully atomistic profile, as observed above in Sec. IV A 3 for the weakest coupling $(\kappa=1 / 3)$. At the heaviest load examined $\left(T_{y y}=-0.3\right)$ the hysteresis loop of the fully atomistic profile extends over virtually the whole range of registers considered, whereas the loop of HACG profile is restricted to the range $0.1<\alpha<0.9$. Nevertheless, the effective thermal elastic coefficients associated with the very narrow nonhysteretic elastic regions of the profiles are in excellent agreement.

\section{B. Multilayer films}

Figure 6 displays shear-stress profiles for multilayer films at $T=0.1, \kappa=1 / 3$ and the relatively heavy load $T_{y y}=$ -0.5 . Comparing HACG with exact profiles, we find excellent agreement in the elastic regions, and, although there are discrepancies in the transition region due to hysteresis, the agreement there is nevertheless quite good. The effective thermal elastic coefficient decreases monotonically as $n_{l}$ increases. This trend is in accord with intuition. Since the interatomic forces within the film are weaker than those within the substrates, the "sandwich" should become weaker as the proportion of film constituting it increases.

Figure 7 shows the effect of lightening the load. We note that at loads lighter than $T_{y y}=-0.3$ a film consisting of more than two layers becomes gaslike and the shear-stress profile flattens. The accuracy of the computed shear stress decreases markedly. This tendency can be seen already in Fig. 7. The profile for the one-layer film exhibits clearly the snapping character: a linear rise in $T_{y x}$ followed by an abrupt change in sign at a critical register [about $\alpha=0.55$ in Fig. 7(a)]. However, as $n_{l}$ increases, the elastic region narrows while the transition region broadens. The four-layer profile is essentially flat. The film behaves as a fluid. It is noteworthy that the HACG profiles agree well overall with the fully atomistic ones.

Comparison of the corresponding shear-stress profiles in Figs. 6 and 7 reveals that the thermal elastic coefficient increases with increasing load, as one would expect. When the
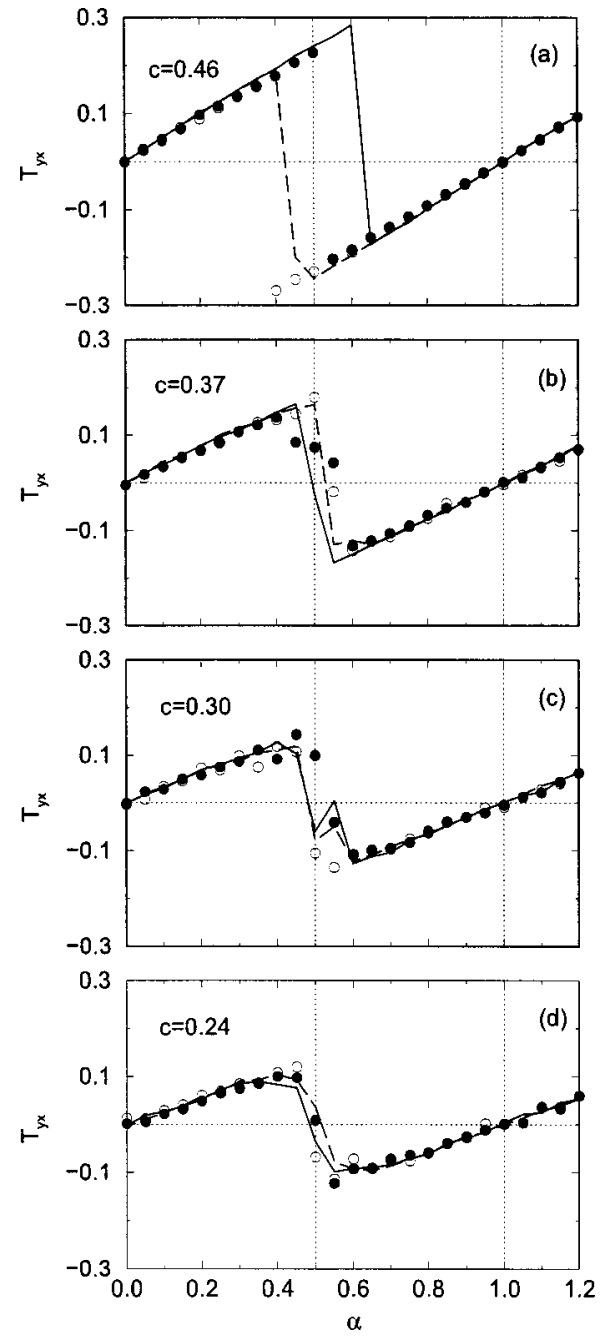

FIG. 6. Shear-stress profiles for model contact with one-layer (a), two-layer (b), three-layer (c), and four-layer (d) film at $T=0.1, T_{y y}=-0.5$, and $\kappa=1 / 3$. Notation as in Fig. 2 .

load is lighter, the film is less compressed and hence the intermolecular forces that oppose the relative sliding of layers are weaker.

The degree of hysteresis, as indicated by the breadth of the loop, appears to decrease markedly as the film thickens. The broadest loop occurs for the monolayer. The barrier to migration of film atoms is greatest in the case of the monolayer because the film atoms are most severely confined to their effective cages.

\section{Role of elasticity of substrates}

We turn now to the results of a systematic study designed to determine the importance of a proper accounting for the elasticity of the substrates. We examined a sequence of model substrates in which the near region is successively enlarged as follows: zero layers of solid atoms [i.e., wholly rigid substrates, as shown in Fig. 1(c)]; 4 layers [Fig. 1(d)]; 8 layers; 12 layers; 16 layers; 24 layers [fully atomistic, Fig. 1(a)]. All models in this succession were simulated atomistically.

Figure 8 compares shear-stress profiles for multilayer films between wholly rigid substrates, substrates with four- 

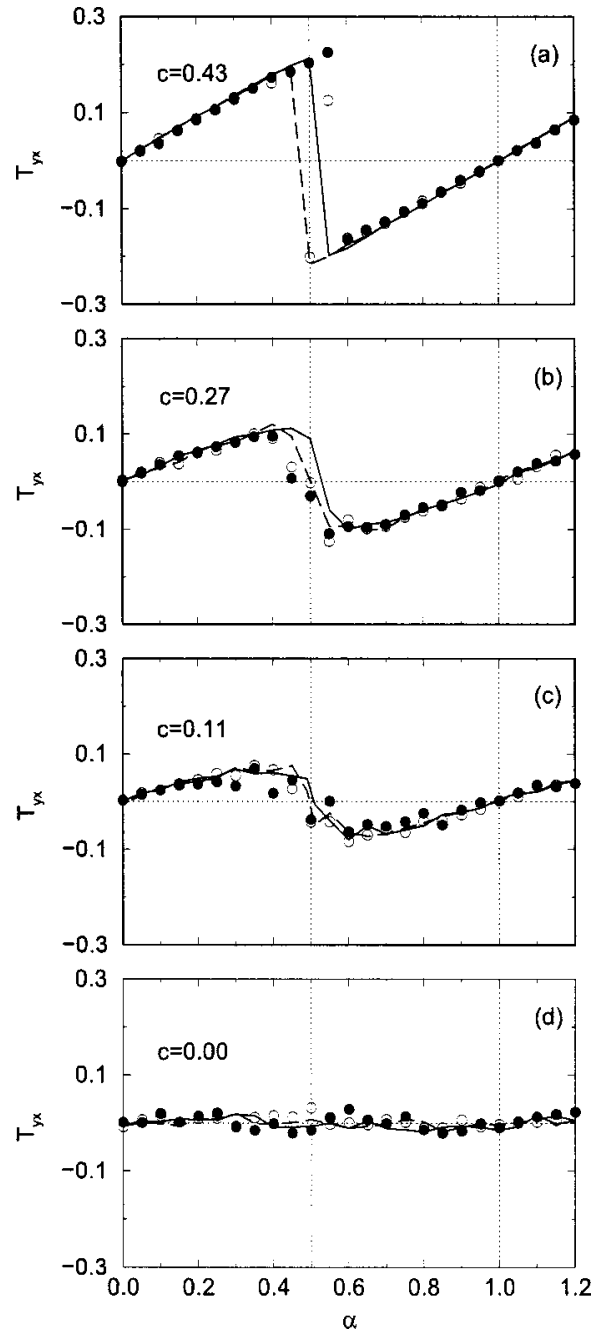

FIG. 7. As Fig. 6, except $T_{y y}=-0.3$.

layer near regions and fully atomistic substrates. The wholly rigid profiles exhibit no snapping character at all. However, as indicated by the initial slope, the thermal elastic coefficient decreases monotonously as $n_{l}$ increases. Also, the maximum shifts to higher register and falls with increasing $n_{l}$. These observations can be readily rationalized. As the film becomes thicker, the template effect becomes weaker. Hence, the thicker the film is, the "softer" it is and the less able it is to sustain a shear stress. These remarks apply as well to the case of substrates with atomistic four-layer near regions [Fig. 1(d)].

Compared with the fully atomistic shear-stress profiles, the wholly rigid and four-layer near-region profiles display no hysteresis (Fig. 8). This may reflect that there are fewer (stochastic) degrees of freedom in the substrates for the latter two versions of the model contact.

We now gradually introduce more elasticity into the substrates using 8-, 12-, 16-, and 24-layer (fully atomistic) near regions. Rather than present shear-stress profiles for all cases at fixed load, we instead look at the maximum magnitude of $T_{y x}$ versus $\alpha\left(\alpha^{\prime}\right)$, which we identify with the static friction. That is, we take the static friction to be equal to the maximum magnitude of $T_{y x}$ multiplied by the length of the contact. This is the minimum force that needs to be applied to
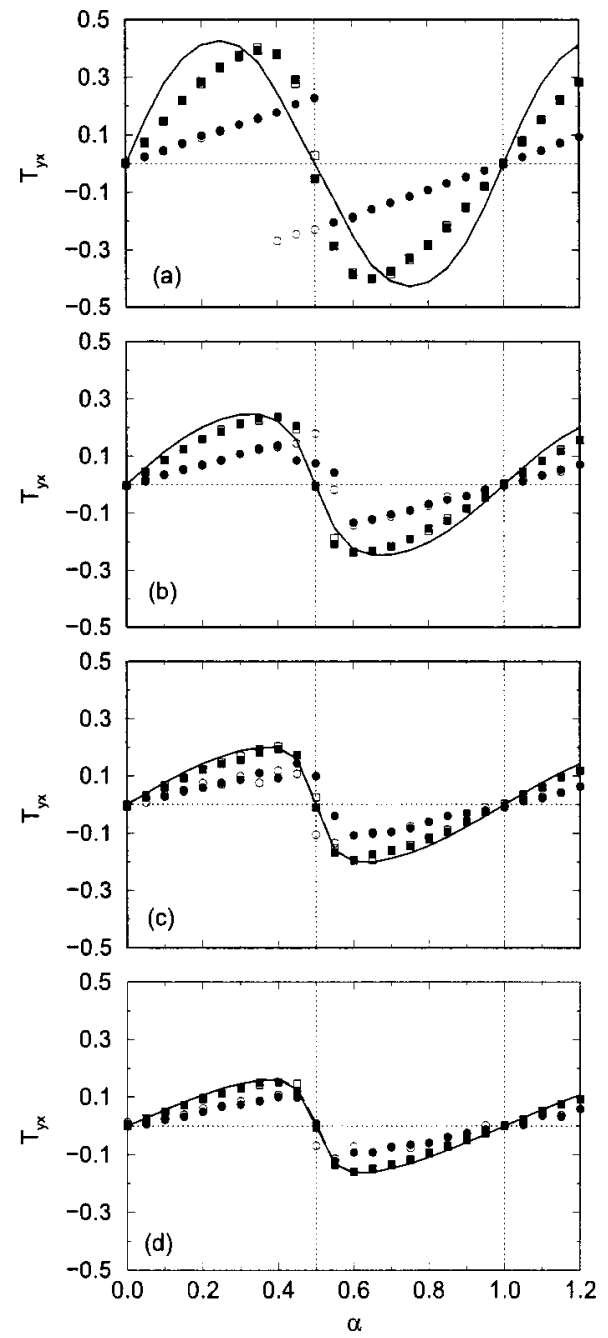

FIG. 8. Shear-stress profiles for model contact with one-layer (a), two-layer (b), three-layer (c), and four-layer (d) film at $T=0.1, T_{y y}=-0.5$, and $\kappa=1 / 3$. Wholly rigid substrates [Fig. 1(c)] (forward, solid line; reverse, dashed line); atomistic four-layer near region [Fig. 1(d)] (forward, filled square; reverse, open square); fully atomistic [Fig. 1(a)] (forward, filled circle; reverse, open circle).

initiate (irreversible) sliding. In Fig. 9 we plot the maximum of $T_{y x}$ versus the load ( $-T_{y y}$ ) for the two-layer film between the successive model substrates described above. As expected, the friction rises monotonously with the load for the same reason that the thermal elastic coefficient increases

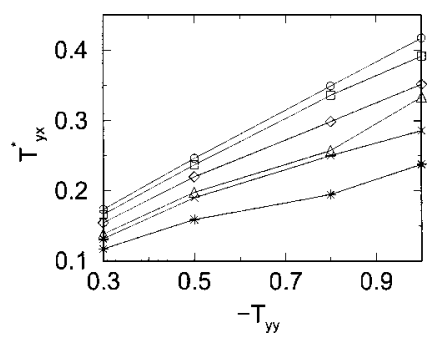

FIG. 9. Friction profiles (maximum magnitude of shear stress $T_{y x}^{*}$ vs $-T_{y y}$ ) for model contact with two-layer film. Wholly rigid substrates [Fig. 1(c)] (circle); 4-layer near region [Fig. 1(d)] (square); 8-layer near region (diamond); 12-layer near region (triangle); 16-layer near region $(\times)$; fully atomistic [Fig. 1(a)] (*). 
with load: the heavier the load, the more compressed the film and consequently the stronger are the intermolecular forces that oppose sliding and eventual snapping of the film.

The friction profile drops uniformly (in $-T_{y y}$ ) as the soft near region extends. We also observe that the profile departs from linearity progressively as the soft near region becomes larger. We speculate that this tendency reflects the increasing number of stochastic variables and the consequent increase in the degree of hysteresis in the transition region. The same effect is noticed in the shear-stress profiles (see Figs. 6 and 7). The monotonic decrease of static friction with increasing size of the soft near region at fixed load is intuitively reasonable. As the substrates become softer, the effective mobility of the film atoms increases, since the solid atoms are able to move out of their way. Therefore the maximum shear stress that the contact can sustain decreases.

\section{CONCLUSION}

This pair of papers (Ref. 21 and the present one) deal with the formulation and testing of the HACG treatment of reversible processes in multiple scale systems with fluidsolid interfaces. Implementing the HACG formalism through an isothermal-isobaric Monte Carlo procedure, we investigated the quasistatic shearing of a 2D model lubricated contact comprising two planar solid Lennard-Jones $(12,6)$ substrates separated by a softer Lennard-Jones $(12,6)$ film. We compared HACG shear-stress profiles (plots of shear-stress versus register) with the fully atomistic ones for several cuts through the four-dimensional thermodynamic-state space characterized by temperature, normal load, film-substrate coupling strength, and film thickness.

The shear-stress profile exhibits symmetries that are explicable in terms of the symmetry inherent in the atomic structure of the substrates. It is periodic in the register, having period unity. A single period of the profile consists of two elastic regions separated by a transition region. In the elastic regions the contact acts as a linear elastic solid. In the transition region it evinces a "snapping" behavior (i.e., at a critical register the substrates tend to slip abruptly past each other).

In the transition region the system exhibits a hysteresis loop whose breadth depends strongly on the thermodynamic state. Hysteresis is especially prominent for the monlayer film, in which case we ascribe it to potential-energy barriers to the migration of film molecules among the effective cages formed by substrate atoms at the interface. As the register goes from integral toward half integral in a given period, the cages become increasingly constricted and the barriers rise accordingly. Hysteresis is much less severe for multilayer films, where predominant film-film interactions are weaker than film-substrate interactions and the barrier to migration is consequently lower. Hysteresis is exacerbated by decreasing the temperature or increasing the load or film-substrate coupling constant.

The HACG and fully atomistic profiles are in excellent agreement in the elastic regions of the shear-stress profiles for the thermodynamic states investigated. They are also in very good agreement in the usually hysteretic transition re- gion. We conclude that the HACG technique furnishes a reliable description of shearing under a wide variety of conditions.

Defining a sequence of model substrates in which the dynamic (atomistic) near region is successively enlarged while the complementary far region is kept rigid, we studied systematically the impact of elasticity of the substrates on the shearing behavior of the contact. As the size of the soft near regions increases, the friction profile (plot of maximum magnitude of $T_{y x}$ versus $-T_{y y}$ ) only gradually homes in on the fully atomistic profile, with which the HACG profile agrees. Thus, an accurate description of sliding cannot be achieved simply by taking a limited atomistic near region and neglecting the elasticity of the far region. The elastic response of the whole substrate must somehow be properly taken into account and this is effectively and efficiently accomplished by the HACG technique.

The HACG scheme, which can be regarded as an extension of the QC treatment of the quasistatic evolution of defects in solids, ${ }^{3}$ has two main new features. The first is that the HACG procedure coarse grains only the remote regions of the solid substrates rather than the whole solid. The second is that the HACG procedure minimizes the free energy of the system rather than the potential energy. In other words, the HACG method is not restricted to zero temperature, as is the original QC technique. It should be noted, however, that the HACG treatment, as presently formulated, suppresses the random thermal motion of all far-region atoms except the nodes. Therefore, it is not obvious that the HACG shearstress profiles should be in as good agreement with the exact (fully atomistic) ones as they are found to be. Indeed, earlier studies $^{23,24}$ showing that dynamically constrained coarse graining can give rise to a substantial error in the isotropic stress of a pure single crystal led us to expect errors of similar magnitude in the HACG sheer stress. Evidently the impact of the neglected thermal motion on shearing is negligible.

The purpose of this work is to formulate and test a method of handling reversible processes in multiple-scale systems where fluid-solid interfaces play the central role. For the sake of computational economy we restrict our attention to a $2 \mathrm{D}$ version of a model, namely, a thin fluid film confined between planar solid substrates, which has been repeatedly investigated since the early 1980's. These have been reviewed several times. ${ }^{25-27}$ Although our principal objective is not to discover a new physics, but rather to validate the HACG scheme, nevertheless the study does reveal an important effect: the crucial impact of the elastic response of the far regions on thermomechanical properties. This is particular evident in the friction profiles of Fig. 9, which have important implications for 3D studies, most of which treat the substrates as wholly rigid.

\section{ACKNOWLEDGMENTS}

We thank the National Science Foundation for generous support through the Grants Nos. ECS-9900127, ITR0112929, ITR-0427746 and NIRT-0210850. We also thank 
the UNL research computing facility and Nebraska Research Initiative for support. We are grateful to Professor Ruqiang Feng for valuable discussions.

${ }^{1}$ S. Kohlhoff, P. Gumbsch, and H. F. Fischmeister, Philos. Mag. A 64, 851 (1991).

${ }^{2}$ P. Gumbsch and G. E. Beltz, Modell. Simul. Mater. Sci. Eng. 3, 597 (1995).

${ }^{3}$ E. B. Tadmor, M. Ortiz, and R. Phillips, Philos. Mag. A 73, 1529 (1996).

${ }^{4}$ E. B. Tadmor, R. Phillips, and M. Ortiz, Langmuir 12, 5429 (1996).

${ }^{5}$ O. A. Shenderova, D. W. Brenner, A. A. Nazarov, A. E. Romanov, and L. H. Yang, Phys. Rev. B 57, R3181 (1998).

${ }^{6}$ H. Rafii-Tabar, L. Hua, and M. Cross, J. Phys.: Condens. Matter 10, 2375 (1998).

${ }^{7}$ E. B. Tadmor, G. S. Smith, N. Bernstein, and E. Kaxiris, Phys. Rev. B 59, 235 (1998)

${ }^{8}$ R. E. Rudd and J. Q. Broughton, Phys. Rev. B 58, R5893 (1998).

${ }^{9}$ F. F. Abraham, J. Q. Broughton, N. Bernstein, and E. Kaxiris, Comput. Phys. 12, 538 (1998).

${ }^{10}$ J. Q. Broughton, F. F. Abraham, N. Bernstein, and E. Kaxiris, Phys. Rev. B 60, 2391 (1999).

${ }^{11}$ R. E. Rudd and J. Q. Broughton, Phys. Status Solidi B 217, 251 (2000).

${ }^{12}$ R. C. Picu, J. Comput.-Aided Mater. Des. 7, 77 (2000).

${ }^{13}$ A. M. Cuitino, L. Stainier, G. Wang, A. Strachan, T. Cagin, W. A. Goddard
III, and M. Ortiz, J. Comput.-Aided Mater. Des. 8, 127 (2001).

${ }^{14}$ S. Curtarolo and G. Ceder, Phys. Rev. Lett. 88, 255504 (2002).

${ }^{15}$ R. Phillips, M. Dittrich, and K. Schulten, Annu. Rev. Mater. Res. 32, 219 (2002).

${ }^{16}$ R. E. Miller and E. B. Tadmor, J. Comput.-Aided Mater. Des. 9, 203 (2002).

${ }^{17}$ W. A. Curtin and R. E. Miller, Modell. Simul. Mater. Sci. Eng. 11, R33 (2003).

${ }^{18}$ R. E. Miller, Int. J. Multiscale Comput. Eng. 1, 57 (2003).

${ }^{19}$ V. B. Shenoy, Bull. Mater. Sci. 26, 53 (2003).

${ }^{20} \mathrm{O}$. C. Zienkiewicz and R. L. Taylor, The Finite Element Method, 5th ed. (Butterworth-Heinemann, Oxford, 2000), Vol. 1, Chap. 4.

${ }^{21}$ Z.-B. Wu, D. J. Diestler, R. Feng, and X. C. Zeng, J. Chem. Phys. 120, 6744 (2004)

${ }^{22}$ C. L. Rhykerd, Jr., M. Schoen, D. J. Diestler, and J. H. Cushman, Nature (London) 330, 461 (1978).

${ }^{23}$ Z.-B. Wu, D. J. Diestler, R. Feng, and X. C. Zeng, J. Chem. Phys. 119, 8013 (2003).

${ }^{24}$ D. J. Diestler, Phys. Rev. B 66, 184104 (2002).

${ }^{25}$ B. Bhushan, J. N. Israelachvili, and U. Landman, Nature (London) 374, 607 (1995).

${ }^{26}$ M. H. Mueser, M. Urbakh, and M. O. Robbins, Adv. Chem. Phys. 126, 187 (2003).

${ }^{27}$ M. O. Robbins and M. H. Mueser, in Modern Tribology Handbook, edited by B. Bhushan (CRC, Boca Raton, 2001), Vol. 1, p. 717. 